\title{
Correlation between Serum Carcinoembryonic Antigen Level and Histologic Subtype in Resected Lung Adenocarcinoma
}

\author{
Masaki Tomita*, Takanori Ayabe, Eiichi Chosa Kunihide Nakamura
}

\begin{abstract}
Background: Recent studies revealed a relationship between ground-glass opacity (GGO) ratio on computed tomography (CT) and serum carcinoembryonic antigen (CEA) level in lung adenocarcinoma. Since an association between lepidic histologic pattern and GGO is well accepted, we investigated the link between histologic subtype and serum CEA level in resected lung adenocarcinoma. Materials and Methods: One hundred and eighty-one consecutive patients with resected lung adenocarcinoma were studied retrospectively. The histologic subtype was subdivided into 2 groups: lepidic dominant histologic subtype, including adenocarcinoma in situ, minimally invasive adenocarcinoma and lepidic predominant invasive adenocarcinoma versus other subtypes. Results: The 5-year survival of patients with $s$ high serum CEA level was significantly more unfavorable than that with normal levels. Similarly, there was also a relationship between the patient survival and histologic subtype, with favorable survival found in patients with the lepidic dominant histologic subtype. There was a significant relationship between serum CEA level and lepidic dominant histologic subtype overall and in p-stage I patients. Conclusions: Lung adenocarcinomas with non-lepidic dominant histologic subtype are associated with high serum CEA levels.
\end{abstract}

Keywords: CEA - lepidic histologic subtype - GGO - lung adenocarcinoma - prognosis

Asian Pac J Cancer Prev, 16 (9), 3857-3860

\section{Introduction}

Several studies revealed that preoperative serum carcinoembryonic antigen (CEA) levels have been reported to be an independent prognostic factor in primary non-small-cell lung cancer (NSCLC) (Okada et al., 2004; Sawabata et al., 2004; Tomita et al., 2004). Therefore high serum CEA level is thought to have much more aggressive biologic features resulting in unfavorable prognosis.

The recent development of high-resolution computed tomography (HRCT) and low-dose computed tomography (CT) screening has improved the detection of small lung cancers, especially lung adenocarcinomas (Shimizu et al., 2005, Kodama et al., 2001, Miao et al., 2012). These often contain a non-solid component that presents as a ground-glass opacity (GGO). GGO is characterized by a lepidic pattern of cells that line the alveoli but do not invade neighboring structures (Kodama et al., 2001; Shimizu et al., 2005; Miao et al., 2012). Previous studies have also shown the proportion of GGO is strongly related to biological malignancy of small adenocarcinoma, and the prognostic value of GGO has been reported (Kodama et al., 2001; Shimizu et al., 2005; Miao et al., 2012). Furthermore recent study indicated the relationship between serum CEA level and CT features, including GGO ratio (Yamazaki et al., 2014).

Since the association between histologic lepidic pattern and GGO has been well accepted (AmbrosiniSpaltro et al., 2014), in the present study, we examined the relationship between serum CEA level and lepidic histologic subtype in lung adenocarcinoma.

\section{Materials and Methods}

One hundred and eighty-one consecutive lung adenocarcinoma patients who underwent surgery from 2007 to 2012 in our hospital were enrolled into the present retrospective study. Patients who did not undergo complete resection, died of other diseases after surgery or were lost to follow-up were excluded.The preoperative serum CEA level was measured using the two-site immunoenzymometric assay; the normal upper limit for this assay was $5.0 \mathrm{ng} / \mathrm{mL}$. Surgical samples were analyzed for epidermal growth factor receptor (EGFR) mutation using Cycleave polymerase chain reaction (PCR) method by SRL Inc. (Tokyo, Japan) (Yatabe et al., 2006). Pathological (p) tumor-node-metastasis (TNM) staging was recorded in all patients based on the 7th edition of the American Joint Committee on Cancer (AJCC)/ Union for International Cancer Control (UICC) classification. Histologic subtype was also recorded based on International association for the study of lung cancer/american thoracic society/european respiratory society international multidisciplinary classification of 


\section{Masaki Tomita et al}

lung adenocarcinoma (Travis et al., 2011). According to the previous studies (Kadota et al., 2014; Weichert et al., 2014), the histologic subtype was subdivided into 2 groups: lepidic dominant histologic subtype, including adenocarcinoma in situ, minimally invasive adenocarcinoma, and lepidic predominant invasive adenocarcinoma versus other subtypes.

Follow-up information, including cause of death, was ascertained through a review of clinic notes and direct or family contact. The survival curves of the patients were plotted by using the Kaplan-Meier method and analyzed using the log-rank test. The chi-square test with Yates' correction was applied to test any association between serum CEA level and cliniopathological factors. The value of serum CEA level was compared based on histologic subtype using students' t-test. Statistical calculations were conducted with JMP (SAS Institute Inc., Cary, NC, USA) and values of $p$ less than 0.05 were accepted as being significant.

\section{Results}

Figure 1A shows the patients' survival based on serum CEA level in overall patients. The 5-year survival of patients with high serum CEA level was $65.41 \%$, which was significantly unfavorable than that with normal serum level $(87.2 \%, \mathrm{p}=0.0045)$. Similarly, as shown in Figure $1 \mathrm{~B}$, there was also a relationship between the patients' survival and histologic subtype, with favorable survival found in patients with lepidic dominant histologic subtype $(\mathrm{p}=0.0045)$.

Table 1 shows the relationship between serum CEA level and clinicopathological factors in overall patients.
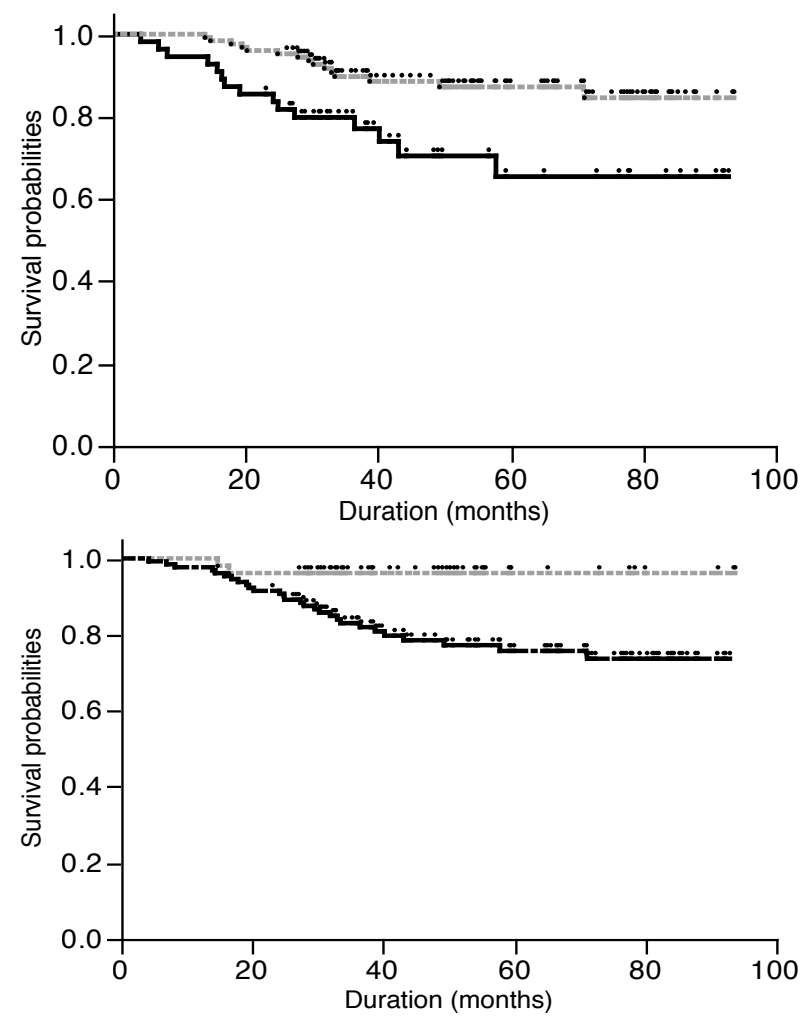

Figure 1. Survival of Patients Based on serum CEA level (A) and Histologic Subtype (B) In Overall Patients
Serum CEA level was significantly associated with gender, p-stage, pT status, pN status, smoking, lepidic dominant histologic subtype and pure/mixed GGO in overall patients. On the other hand, age and EGFR mutation status were not.

Since serum CEA level was related to $\mathrm{p}$-stage and $\mathrm{pN}$ status in overall patients, we also examined the patients with $\mathrm{p}$-stage I diseases $(n=128)$ to exclude the effect of p-stage and $\mathrm{pN}$ factors. As shown in Figure 2, p-stage I

Table 1. Comparison of Clinical Characteristics Based on Serum CEA Level in Overall Patients

\begin{tabular}{llccc}
\hline & & $5>$ CEA & $5 \leq$ CEA & p value \\
\hline Age & $65<$ & 47 & 20 & 0.9043 \\
& $65 \geq$ & 79 & 35 & \\
Gender & Male & 54 & 34 & 0.0185 \\
& Female & 72 & 21 & \\
p-stage & I & 100 & 28 & 0.0002 \\
& II-III & 26 & 27 & \\
pT status & pT1 & 90 & 30 & 0.0289 \\
& pT2-3 & 36 & 25 & \\
pN status & pN0 & 108 & 35 & 0.0011 \\
& pN1-2 & 18 & 20 & \\
Smoking & Never & 71 & 19 & 0.0066 \\
& Current/former & 55 & 36 & \\
EGFR & Wild type & 60 & 32 & 0.1903 \\
& Mutant EGFR & 66 & 23 & \\
Histologic- & Lepidic dominant & 44 & 8 & 0.0037 \\
subtype & Others & 82 & 47 & \\
GGO & Pure/mixed GGN & 55 & 15 & 0.0347 \\
& Solid & 71 & 40 & \\
\hline
\end{tabular}

*CEA: carcinoembryonic antigen, GGO: ground-glass opacity, EGFR: Epidermal growth factor receptor
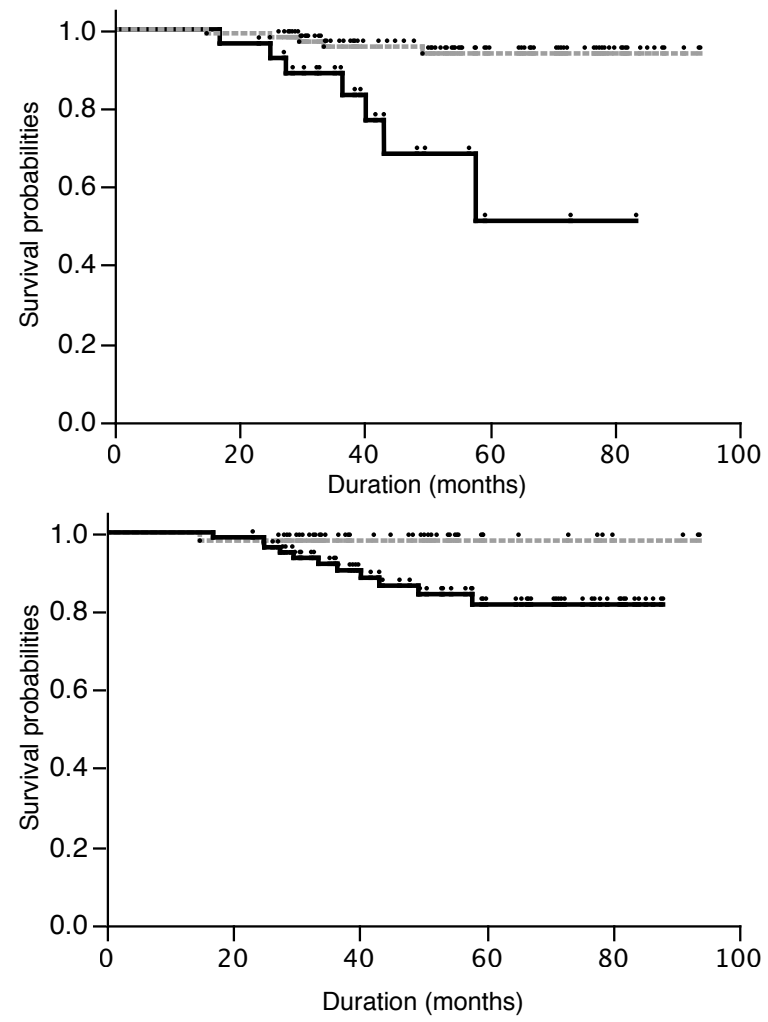

Figure 2. Survival of Patients Based on Serum CEA Level (A) and Histologic Subtype (B) in p-stage I Patients 
Table 2. Comparison of Clinical Characteristics Based on Serum CEA Level in Patients with p-stage I Disease

\begin{tabular}{llccc}
\hline & & $5>$ CEA & $5 \leq$ CEA & p value \\
\hline Age & $65<$ & 24 & 69 & 0.0654 \\
& $65 \geq$ & 4 & 31 & \\
Gender & Male & 17 & 39 & 0.0411 \\
& Female & 11 & 61 & \\
pT status & pT1 & 20 & 85 & 0.1131 \\
& pT2-3 & 8 & 15 & \\
Smoking & Never & 18 & 41 & 0.0286 \\
& Current/former & 10 & 59 & \\
EGFR & Wild type & 13 & 59 & 0.2375 \\
& Mutant EGFR & 15 & 41 & \\
Histologic- & Lepidic dominant & 5 & 43 & 0.0113 \\
subtype & Others & 23 & 57 & \\
GGO & Pure/mixed GGN & 11 & 53 & 0.1982 \\
& Solid & 17 & 47 & \\
\hline
\end{tabular}

*CEA: carcinoembryonic antigen, GGO: ground-glass opacity, EGFR: Epidermal growth factor receptor

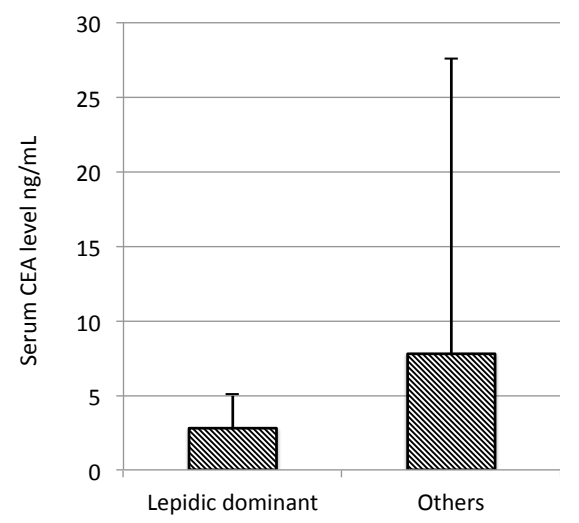

Figure 3. Comparison of the serum CEA value based on histologic subtype

patients with normal serum CEA level had a better 5-year survival rate $(93.87 \%$ vs $51.26 \%, \mathrm{p}=0.0003)$. Similarly the 5-year survival rates of patients with lepidic dominant histologic subtype and others were $97.92 \%$ and $81.65 \%$, respectively $(\mathrm{p}=0.047)$. In patients with $\mathrm{p}$-stage I disease, serum CEA level was also significantly associated with gender, smoking and lepidic dominant histologic subtype but not age, pT status, EGFR mutation status and pure/ mixed GGO (Table 2). The value of serum CEA level was also compared based on histologic subtype in patients with p-stage I disease. As shown in Figure 3, serum CEA level of patients with lepidic dominant histologic subtype was $2.80 \pm 2.20$, while that of patients with others was $7.78 \pm$ $19.87(\mathrm{p}=0.0145)$.

\section{Discussion}

CEA is one of the tumor markers widely used in clinic that can be directly produced by tumor cells and is in close association with patients' prognosis (Okada et al., 2004; Sawabata et al., 2004; Tomita et al., 2004). It can also be secreted by the expression of tumor cell genes to early diagnose lung cancer (Wang et al., 2013; Wang et al., 2014a; 2014b), evaluate therapeutic effect and observe the progression of disease (Qin et al., 2013). In the present study, lung adenocarcinoma patients with high serum CEA level had unfavorable prognosis in overall and p-stage I disease, in agreement with the results of previous studies (Okada et al., 2004; Sawabata et al., 2004; Tomita et al., 2004). Therefore high serum CEA level is thought to have much more aggressive biologic features. Similarly favorable survival was found in patients with lepidic dominant histologic subtype. This might be due to lepidic dominant lung adenocarcinoma has low incidence of lymphatic vessel invasion (Araki et al., 2014).

Our results showed that serum CEA level was related to $\mathrm{p}$-stage and $\mathrm{pN}$ status. Previous studies also reported that serum CEA level is a clinical predictor of $\mathrm{N} 2$ disease (Takamochi et al., 2000; Ye et al., 2014). Therefore unfavorable prognosis in patients with high serum CEA level might be due to higher stage of the diseases at least in part. To avoid the effect of staging, especially N2 disease, we also examined using patients with p-stage I disease. It was found that serum CEA level and lepidic dominant histologic subtype was also related to patients' prognosis in patients with p-stage I disease.

Furthermore we found the relationship between high serum CEA level and non-lepidic dominant histologic subtype in overall and p-stage I lung adenocarcinoma. The exact mechanism leading to high serum CEA levels in patients with NSCLC remains uncertain. However, in colorectal cancer, some possible roles of CEA had been reported. Among these, the degree of local tumor invasion has been considered to play an important role in the increase of serum CEA levels (Zamcheck et al., 1975, Hamada et al., 1985). In NSCLC, it had been reported that high serum CEA levels showed significantly higher rates of pleural invasion and vascular invasion in p-stage I disease (Matsuguma et al., 2008). Therefore it is considered that serum CEA levels in lung adenocarcinoma increase as local tumor invasion progresses. In other word, serum CEA level might be an indicator of the pathological tumor invasion in lung adenocarcinoma.

We also found the relationship between serum CEA level and pure/mixed GGO in overall patients, but failed to find this relationship in p-stage I patients. It was reported that although the percentage of GGO and tumor disappearance rate (TDR) were both associated with the pathological extent of replacement tumor growth, TDR showed a stronger association; the correlation coefficient of TDR was reported to be higher than that of GGO (Okada et al., 2007). Since we did not use TDR, this might be one of reason for our failure to find the relationship between serum CEA level and GGO in p-stage I disease.

With regard to other factors examined, the smoking status and gender were also related to serum CEA level but EGFR mutation status was not. Moreover pT status was related to serum CEA level in overall patients but not patients with p-stage I disease. The influence of smoking on serum CEA level had been well accepted (Fukuda et al., 1998). However, to our knowledge, there are few studies that revealed the relationship between serum CEA level and gender. In our series, there were 83/88 smokers in men, while $8 / 93$ in women. Since the smoking rate is several-fold different between men and women, the variables influenced by smoking may show a seeming gender difference. With regard to EGFR mutation and $\mathrm{pT}$ 
status, our previous studies also showed that serum CEA levels do not always relate to EGFR mutation and pT status (Tomita et al., 2006; Tomita et al., 2013).

In conclusions, lung adenocarcinomas with nonlepidic dominant histologic subtype are associated with high serum CEA levels. Therefore serum CEA level might be an indicator of the pathological tumor invasion in lung adenocarcinoma.

\section{References}

Ambrosini-Spaltro A, Ruiu A, Seebacher C, et al (2014). Impact of the IASLC/ATS/ERS classification in pN0 pulmonary adenocarcinomas: a study with radiological-pathological comparisons and survival analyses. Pathol Res Pract, 210, 40-6.

Araki K, Kidokoro Y, Hosoya K, et al (2014). Excellent prognosis of lepidic-predominant lung adenocarcinoma: low incidence of lymphatic vessel invasion as a key factor. Anticancer Res, 34, 3153-6.

Fukuda I, Yamakado M, Kiyose H (1998). Influence of smoking on serum carcinoembryonic antigen levels in subjects who underwent multiphasic health testing and services. J Med Syst, 22, 89-93.

Hamada Y, Yamamura M, Hioki K, et al (1985). Immunohistochemical study of carcinoembryonic antigen in patients with colorectal cancer. Correlation with plasma carcinoembryonic antigen levels. Cancer, 55, 136-41.

Kadota K, Villena-Vargas J, Yoshizawa A, et al (2014). Prognostic significance of adenocarcinoma in situ, minimally invasive adenocarcinoma, and nonmucinous lepidic predominant invasive adenocarcinoma of the lung in patients with stage I disease. Am J Surg Pathol, 38, 448-60.

Kodama K, Higashiyama M, Yokouchi H, et al (2001). Prognostic value of ground-glass opacity found in small lung adenocarcinoma on high-resolution CT scanning. Lung Cancer, 33, 17-25.

Matsuguma H, Nakahara R, Igarashi S, et al (2008). Pathologic stage I non-small cell lung cancer with high levels of preoperative serum carcinoembryonic antigen: clinicopathologic characteristics and prognosis. $J$ Thorac Cardiovasc Surg, 135, 44-9.

Miao XH, Yao YW, Yuan DM, et al (2012). Prognostic value of the ratio of ground glass opacity on computed tomography in small lung adenocarcinoma: A meta-analysis. $J$ Thorac Dis, 4, 265-71.

Okada M, Nishio W, Sakamoto T, et al (2004). Prognostic significance of perioperative serum carcinoembryonic antigen in non-small cell lung cancer: analysis of 1,000 consecutive resections for clinical stage I disease. Ann Thorac Surg, 78, 216-21.

Okada M, Tauchi S, Iwanaga K, et al (2007). Associations among bronchioloalveolar carcinoma components, positron emission tomographic and computed tomographic findings, and malignant behavior in small lung adenocarcinomas. $J$ Thorac Cardiovasc Surg, 133, 1448-54.

Qin HF, Qu LL, Liu H, et al (2013). Serum CEA level change and its significance before and after Gefitinib therapy on patients with advanced non-small cell lung cancer. Asian Pac J Cancer Prev, 14, 4205-8.

Sawabata N, Maeda H, Yokota S, et al (2004). Postoperative serum carcinoembryonic antigen levels in patients with pathologic stage IA nonsmall cell lung carcinoma: subnormal levels as an indicator of favorable prognosis. Cancer, 101, 803-9.

Shimizu K, Yamada K, Saito H, et al (2005). Surgically curable peripheral lung carcinoma: correlation of thin-section CT findings with histologic prognostic factors and survival. Chest, 127, 871-8.

Takamochi K, Nagai K, Suzuki K, et al (2000). Clinical predictors of $\mathrm{N} 2$ disease in non-small cell lung cancer. Chest, 117, 1577-82.

Tomita M, Ayabe T, Chosa E, et al (2013). Is there a relationship between serum carcinoembryonic antigen level and epidermal growth factor receptor mutations in resected lung adenocarcinomas ? Ann Cancer Res Ther, 21, 31-5.

Tomita M, Matsuzaki Y, Edagawa M, et al (2004). Prognostic significance of preoperative serum carcinoembryonic antigen level in lung adenocarcinoma but not squamous cell carcinoma. Ann Thorac Cardiovasc Surg, 10, 76-80.

Tomita M, Matsuzaki Y, Shimizu T, et al (2006). Relationship between serum carcinoembryonic antigen level and $\mathrm{T}$ status in non-small cell lung cancer. Anticancer Res, 26, 3845-8.

Travis WD, Brambilla E, Noguchi M, et al (2011). International association for the study of lung cancer/american thoracic society/european respiratory society international multidisciplinary classification of lung adenocarcinoma. $J$ Thorac Oncol, 6, 244-85.

Wang B, He YJ, Tian YX, et al (2014). Clinical utility of haptoglobin in combination with CEA, NSE and CYFRA21-1 for diagnosis of lung cancer. Asian Pac J Cancer Prev, 15, 9611-4.

Wang WJ, Tao Z, Gu W, et al (2013). Clinical observations on the association between diagnosis of lung cancer and serum tumor markers in combination. Asian Pac J Cancer Prev, 14, 4369-71.

Wang WT, Li Y, Ma J, et al (2014). Serum carcinoembryonic antigen levels before initial treatment are associated with EGFR mutations and EML4- ALK fusion gene in lung adenocarcinoma patients. Asian Pac J Cancer Prev, 15, 3927-32.

Weichert W, Warth A (2014). Early lung cancer with lepidic pattern: adenocarcinoma in situ, minimally invasive adenocarcinoma, and lepidic predominant adenocarcinoma. Curr Opin Pulm Med, 20, 309-16.

Yamazaki M, Ishikawa H, Kunii R, et al (2014). Relationship between $\mathrm{CT}$ features and high preoperative serum carcinoembryonic antigen levels in early-stage lung adenocarcinoma. Clin Radiol, 69, 559-66.

Yatabe Y, Hida T, Horio Y, et al (2006). A rapid, sensitive assay to detect EGFR mutation in small biopsy specimens from lung cancer. $J$ Mol Diagn, 8, 335-41.

Ye B, Cheng M, Ge XX, et al (2014). Factors that predict lymph node status in clinical stage T1aN0M0 lung adenocarcinomas. World J Surg Oncol, 12, 42.

Zamcheck N, Doos WG, Prudente R, et al (1975). Prognostic factors in colon carcinoma: correlation of serum carcinoembryonic antigen level and tumor histopathology. Hum Pathol, 6, 31-45. 